\title{
Cosmetics and pharmaceutics: new trends in biophysical approaches
}

\author{
Salvatore Magazù
}

(C) European Biophysical Societies' Association 2012

The papers in this Special Issue of European Biophysical Journal are linked to the international conference promoted by LE STUDIUM ${ }^{\circledR}$ (http://lestudium.cnrs-orleans.fr), entitled "Cosmetics and Pharmaceutics: New Trends in Biophysical Approaches" which took place on 14-15 February 2011, in Orléans, in the heart of France, a leading region in cosmetics and pharmaceutics.

As an Institute for Advanced Studies connected to the research institutions located in the Loire Valley (France), LE STUDIUM ${ }^{\circledR}$ wishes to accompany the efforts made by the "Cosmetic Valley" cluster, whose aim is also to develop joint research and industrial approaches in cosmetics and pharmaceutics. The LE STUDIUM ${ }^{\circledR}$ Conference was primarily aimed to help technology transfer from research to the industrial scale and to update knowledge in the fields of biophysics, chemistry and biochemistry applied to cosmetics and pharmaceutics. It gathered renowned academic and industrial experts and offered an extraordinary chance to tackle and debate the latest topics, issues, trends and challenges of the cosmetic and pharmaceutical industry. Special care was taken to facilitate effective dialogue between actors from completely different horizons. In addition to the participation of internationally recognized senior scientists, the conference encouraged participation of young researchers via a devoted poster session. The conference was also supported by the European Biophysical Societies' Association (EBSA, http://www.ebsa.org/) and received the scientific label from the Université Franco Italienne/Università Italo Francese

S. Magazù $(\bowtie)$

Department of Physics, University of Messina,

Viale Ferdinando Stagno D'Alcontres No. 31,

P.O. Box 55, 98166 S. Agata, Messina, Italy

e-mail: smagazu@unime.it
(UIF/UFI, http://www.universite-franco-italienne.org). EBSA bursaries were also available to European students or young postdocs who attended the conference. The scientific program spanned advanced fundamental research fields such as protein denaturation, bioprotective molecular mechanisms, interface chemical processes, physical confinement effects, spectroscopic and simulation techniques, all in the context of application to cosmetics and pharmaceutics. In addition, since the conference was held during the International Year of Chemistry, special emphasis was given to links between biophysics, biochemistry and chemistry. The conference included plenary sessions, oral presentations, poster sessions and a lively round-table. The latter allowed the creation of an ideal atmosphere where internationally competitive researchers and industry representatives shared the common goal of planning and putting into action a series of initiatives addressing promotion of applied research directed towards analysis of some topical themes in cosmetics and pharmaceutics. Here, it was well established that research plays a key role within Cosmetic Valley, and in this framework close interactions from large industrial groups with smaller companies and research teams from universities should be increasingly encouraged.

The papers in this Special Issue report on the LE STUDIUM $^{\circledR}$ Conference contributions, discuss likely directions of future work on cosmetics and pharmaceutics and outline some challenging, hitherto-inaccessible problem areas. This compilation of contributions, which furnishes a representative picture of the new trends in biophysical approaches, can be considered as the harvest of recent efforts to understand the relation between the structure and function of biological systems, which ultimately requires microscopic understanding of the underlying conformational dynamics, and a broad range of 
applications in both the cosmetic and pharmaceutical fields. In this frame, the contributions deal with new biophysical approaches addressed at understanding, at a molecular level, the physico-chemical phenomena of condensed phases and dispersed matter systems which are the basis for formulation and vectorization as well as characterization and optimization of biomarkers and bioactives. The biological structures taken into account are not to be considered as static systems as depicted by Schrodinger's term "aperiodic crystals" but as dynamical systems which perform motions to execute their functions. These motions involve atoms not just of the biological structure itself but also of the surrounding medium with which a coupling exists, as characterized by an energy landscape organized in hierarchies with valleys and within valleys, in a series of tiers where transition among substrates gives rise to motions which are essential for function. The articles in this Special Issue refer to experimental, theoretical, numerical and technical studies: privileged partners in the scientific endeavour to understand at a molecular level such complex systems. They include experimental studies using a wide variety of techniques, theoretical and numerical investigations and innovative sample environment devices. The articles highlight the dynamic richness of biophysical systems, which often require the application of different, complementary techniques to be disentangled. Studies of the coupling between solvent dynamics and biostructure dynamics, from small molecules to macromolecules, are a good example of this. They provide new insights into how biomolecular dynamics and function can be controlled by a properly chosen environment. In a symmetric way, these studies also enrich our knowledge of the behaviour of water in the presence of biomolecules, and clarify the roles of tetrahedral organization, preferential exclusion, the solvent-protein interface, confinement etc.

In cosmetics and pharmaceutics, innovation exhibits rapid turnover, and only a structured research approach can allow the development of new products that answer market needs while integrating the latest technological discoveries. More than ever before, the promising application of biophysical approaches to the ever-widening field of cosmetics and pharmaceutics is exciting. 